\title{
Excitable Optical Waves in Semiconductor Microcavities
}

\author{
Francesco Marino and Salvador Balle \\ Institut Mediterrani d'Estudis Avançats, CSIC-UIB, E-07071 Palma de Mallorca, Spain
}

(Received 7 June 2004; published 9 March 2005)

\begin{abstract}
We demonstrate experimentally and theoretically the existence of excitable optical waves in semiconductor microcavities. Although similar to those observed in biological and chemical systems, these excitable optical waves are self-confined. This is due to a new dynamical scenario, where a stationary Turing pattern controls the propagation of waves in an excitable medium, thus bringing together the two paradigms of dynamical behavior (waves and patterns) in active media.
\end{abstract}

DOI: 10.1103/PhysRevLett.94.094101

PACS numbers: 05.45.-a, 42.65.Sf, 42.65.Tg

Excitability is a general behavior encountered in many fields of science including biology, chemistry, and physics [1]. Excitable systems have one stable steady state and a thresholdlike response to external perturbations: for stimuli above a certain threshold the system recovers its stable state by emitting a pulse of well defined amplitude and duration (refractory time), independent of the details of the perturbation. In spatially extended excitable media where diffusion processes occur, complex spatiotemporal dynamics arise, and propagating pulses, target waves [2], spiral waves [3], or stationary Turing patterns [4] have been observed. Excitable waves are the result of the propagation of a locally induced nonlinear response through the whole system, while Turing patterns are stationary heterogeneous spatial states arising from spontaneous symmetry-breaking phenomena [5]. These spatiotemporal dynamics play an important role in the functional aspects of many biological systems [6,7]; for instance, they constitute the main mechanism of signal propagation in nerve and cardiac cells [8-10]. Until now, excitable waves have been mainly studied in biological and chemical systems [11], although their existence in optics has been theoretically predicted in several systems [12-14].

It has recently been shown that semiconductor microcavities with optical injection may exhibit global excitable behavior $[15,16]$ compatible with the scenario of the Fitzhugh-Nagumo model [17]. However, due to the small transverse dimensions of the device, no propagation phenomena have been observed. Vertical-cavity semiconductor optical amplifiers (VCSOAs) allow us to make the passage from an excitable system to a $2 \mathrm{D}$ excitable medium. VCSOAs have a short cavity length $(\approx 1 \mu \mathrm{m})$ and a short active medium that ensure longitudinal correlation in the system, but their transverse dimension $\delta$ can be quite large. In this case, the eventual existence of an excitable regime together with carrier diffusion give the necessary conditions for the existence of excitable optical waves propagating through the transverse plane. In broad-area devices $(\delta \geq 50 \mu \mathrm{m})$, though, a homogeneous injected beam can yield a nonhomogeneous output because of a Turing instability of the optical field $[18,19]$. In these conditions, several spatial structures like rings, rolls, and hexagonal patterns [18], and even the formation of cavity solitons $[19,20]$, can be found depending on the system parameters. These patterns break the homogeneity of the excitable medium, thus they may affect the propagation of excitable waves.

Here we report the first experimental observation of excitable optical waves in semiconductor microcavities. Although similar to those observed in biological and chemical systems, these excitable optical waves are selfconfined. This is due to a new dynamical scenario, where a stationary Turing pattern controls the propagation of waves in an excitable medium, thus bringing together the two paradigms of dynamical behavior (waves and patterns) in active media. It has recently been predicted that the two

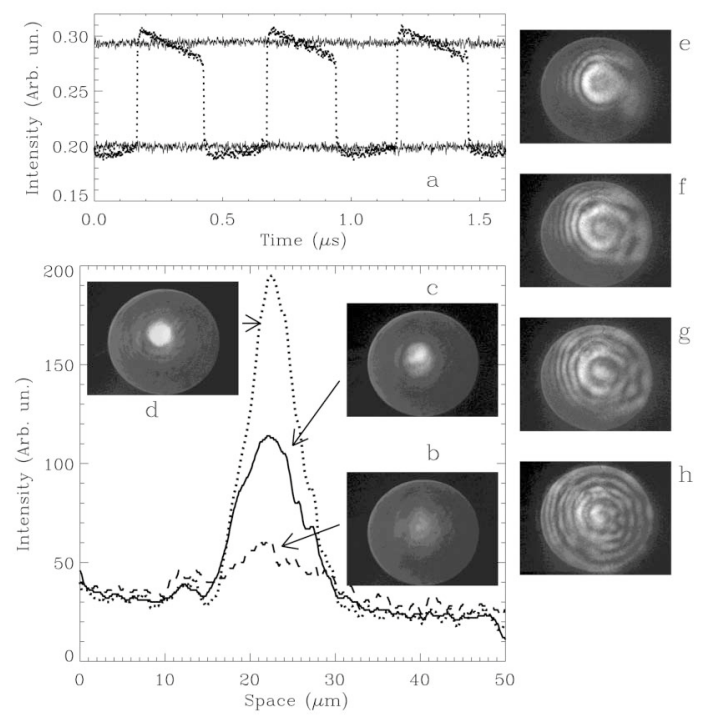

FIG. 1. (a) Time traces corresponding to the following states: lower and upper state (solid traces) and self-oscillating state (dotted trace). Below: transverse cuts of the amplifier output and their corresponding near field CCD images in the (b) low-power state (bias current $177.2 \mathrm{~mA}$ ), (c) self-oscillating state (bias current $177.6 \mathrm{~mA}$ ), and (d) high-power state (bias current $178.1 \mathrm{~mA}$ ). (e)-(h) Near field CCD images for current values: $179.6,183.1,187.9$, and $192.6 \mathrm{~mA}$. The injected wavelength is $982.1 \mathrm{~nm}$ and the injected power is $1 \mathrm{~mW}$. 
phenomena may appear simultaneously [21], but no observations of such a behavior have been reported. We also show that a physical model of our system reproduces the experimental results.

In our experiment we inject monochromatic light into an electrically pumped $54 \mu \mathrm{m}$ oxide-confined VCSOA operating close to $980 \mathrm{~nm}$. The active region consists of three quantum wells $(\mathrm{QW})$ embedded between two Bragg mirrors consisting of $17 n$-type and $30 p$-type pairs. The input beam (diameter $\sim 50 \mu \mathrm{m}$ ) is provided by a tunable external-cavity laser (for details see Ref. [22]). A chargecoupled device (CCD) camera records a time-averaged image of the output facet of the VCSOA, while a fast photodetector is used to monitor the corresponding total output intensity.

By increasing the VCSOA bias current, we pass from a stable state of low output power [lower solid trace in Fig. 1(a)] to a stable state of high output power (higher solid trace) crossing a regime of self-sustained oscillations (dotted trace), typical in FitzHugh-Nagumo systems $[15,16]$. Accompanying this transition, there is a change in the output profile of the system [see Figs. 1(b)-1(d)]: the low-power state is quite homogeneous, but the high-power state displays a bright spot. The image of the selfoscillating regime [Fig. 1(c)] appears as an intermediate situation between the low- and high-power states due to the temporal average performed by the CCD camera, hence revealing that the observed pulsation is not an oscillation of the whole system. This is due to a Turing instability of the optical field in the upper branch, where a localized spatial structure develops [19]. Further increasing the current leads to a pattern of modulated rings whose modulation pitch decreases as the bias current increases [Figs. 1(e)-



FIG. 2. Excitable pulse emitted by the amplifier in response to an external perturbation. The dashed line shows the time when the perturbation is applied. Inset: CCD image of the transverse profile of the output obtained by applying a periodic sequence of fast perturbations (8 ns duration; $100 \mathrm{mV}$ amplitude, corresponding to a bias current variation of $2 \mathrm{~mA} ; 1.0 \mu$ s repetition period). 1(h)] [23]. Although these patterns are influenced by the transverse boundary conditions, they have a nonlinear origin: if the injected optical power is decreased in, e.g., Fig. 1(h), the modulation of the rings disappears.

The same sequence can also be observed by fixing the bias current and decreasing the injected wavelength, which can be understood as the effect of thermal tuning of the cavity resonance due to Joule heating of the VCSOA.

When the system is in its low-power state, excitable upward pulses in the system's response can be triggered by applying fast perturbations to the bias current (see Fig. 2). The response remains spatially confined (see inset), displaying a spot-similar to that of the self-oscillating regime in Fig. 1-which may correspond to a confined target wave, where excitable pulses arise at one spatial point and then they propagate away from it while losing amplitude. In order to check this hypothesis, we simultaneously monitor the output intensity coming from two different points of the VCSOA with two fast avalanche photodetectors (APD), one fixed at the center of the bright spot, while the other can be displaced through the VCSOA transverse plane. The delay between the traces of the APDs increases with the distance between the two monitored points [see Fig. 3(a)], thus confirming our hypothesis. The delay time (at $50 \%$ of the pulse height) is proportional to the distance between observed points in the middle region, but it seems to saturate just before the pulses die [see Fig. 3(b)]. This may indicate a spatial inhomogeneity of the system [24], corroborated by the decrease in the pulse amplitude as the monitored points get further away. For higher injected power, the self-confined excitable optical waves disappear and bistability between the homogeneous low-power state and a localized structure is observed. This is one of the characteristics of cavity solitons recently observed in these devices [20]. Then, the

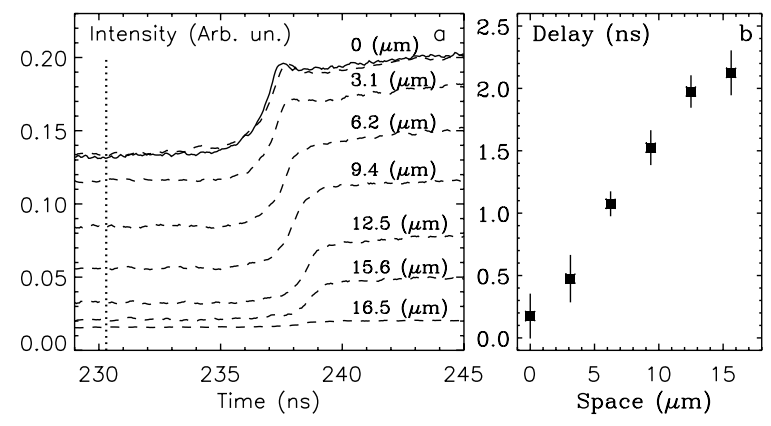

FIG. 3. (a) Rising edge of the excitable pulse in different spatial points after the application of a perturbation to the VCSOA current: the dotted line shows the time when the perturbation is applied, the solid trace is the reference excitable pulse in the center of the bright spot (inset of Fig. 2), and the dashed traces represent the pulse in different spatial points as the distance from the spot center increases. (b) Corresponding time delay at $50 \%$ of the rising edges as a function of distance from the center of the bright spot. 
self-confined excitable waves could be related to the (temperature induced) instability of cavity solitons. These observations have been reproduced in another VCSOA, thus confirming the reproducibility of the experimental results.

Excitable dynamics in semiconductor microcavities arise from the coupling of temperature with the optical and material variables $[15,16]$. In [16], a model was developed for a similar system without spatial degrees of freedom: excitability appears because the interaction between the field and the carriers modifies the power dissipated into the cavity and thus it makes the temperature vary. Temperature variations in turn induce a refractive index change that shifts the cavity resonances, and hence the amount of stimulated emission in the cavity and the dissipated power. This effect may lead to self-oscillations and excitable behavior of the optical power instead of the expected optical bistability [18]. The model in [16] can be generalized by including carrier and thermal diffusions and field diffraction. We consider the injection of a monochromatic field at optical frequency $\omega$ in normal incidence over the first mirror of the VCSOA. We solve the travelingwave equations for the left- and right-propagating fields inside the cavity (length $L$ ), and we impose the boundary conditions at the cavity mirrors and at the QW (located at $z_{0}$ ) in the paraxial approximation. We assume that the field evolves on a time scale much shorter than those for the carrier density and the temperature, so that it adapts instantaneously to the distributions of these two magnitudes. Upon suitable scaling, the resulting model reads

$$
\begin{gathered}
\kappa\left(F+\frac{1}{2 q^{2}} \nabla_{\perp}^{2} F\right)=\frac{1-i \alpha}{2} G F+\mu F_{i}, \\
\partial_{t} G=G_{0}-G-G|F|^{2}+L_{G}^{2} \nabla_{\perp}^{2} G, \\
-\gamma_{t h}^{-1} \partial_{t} q=\left(q-q_{0}-H-L_{t h}^{2} \nabla_{\perp}^{2} q\right),
\end{gathered}
$$

where $F$ is the intracavity optical field at the QW plane, $G$ is the single-pass gain, and $q=n \omega / c$ is the propagation wave vector of the injected field $F_{i}$ inside the cavity. In these equations we have defined

$$
\begin{gathered}
\kappa=\frac{1-r_{1} r_{2} e^{i 2 q L}}{\left(1+r_{1} e^{i 2 q z_{0}}\right)\left[1+r_{2} e^{i 2 q\left(L-z_{0}\right)}\right]}, \\
\mu=\frac{t_{1}^{\prime} e^{i q z_{0}}}{1+r_{1} e^{i 2 q z_{0}}},
\end{gathered}
$$

where $\nabla_{\perp}^{2}=\partial_{x}^{2}+\partial_{y}^{2}$ denotes the transverse Laplacian, $r_{\perp}=(x, y)$, and $G_{0}\left(r_{\perp}\right)$ is the gain distribution due to current injection into the device which we take homogeneous inside a disk of radius $R . L_{G}\left(L_{t h}\right)$ is the diffusion lengths for the carriers (temperature). Finally, $r_{i}\left(r_{i}^{\prime}\right)$ and $t_{i}$ $\left(t_{i}^{\prime}\right)$ denote the internal (external) reflection and transmission coefficients of each cavity mirror. For the sake of simplicity we consider a symmetric VCSOA structure, with identical Bragg mirrors and $z_{0}=L / 2$. This assumption allows us to reduce the number of parameters to be explored, but it does not affect the generality of our results.

In this model, excitability arises from the dynamics of $q$ on the slow thermal time scale $\gamma_{t h}^{-1}$. The dynamical character of $q$ is due to the temperature sensitivity of $n$, assumed to be linear. In the absence of optical field, $q$ would evolve towards a stationary distribution $q_{0}\left(r_{\perp}\right)$, determined both by the Peltier element fixing the temperature of the device's substrate and by Joule dissipation. However, the power locally dissipated in the device increases due to optical injection while it is reduced due to stimulated and spontaneous emission; the contribution of these effects is given by the local energy balance $H=$ $A\left(\left|F_{i}\right|^{2}-\left|F_{r}\right|^{2}-\left|F_{t}\right|^{2}\right)-B G$, where $B G$ is the power emitted by spontaneous emission. $F_{r, t}$ are the reflected
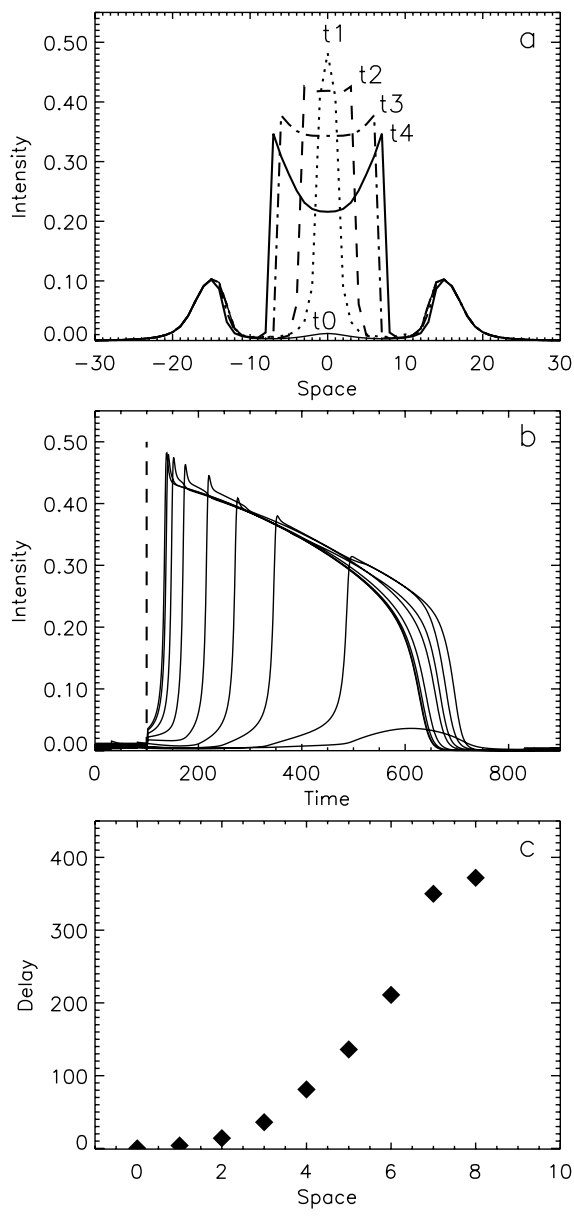

FIG. 4. (a) Transverse cut of the intensity at different times $\left(t_{0}<t_{1}<t_{2}<t_{3}<t_{4}\right)$ after a perturbation is applied. (b) The corresponding excitable response in different positions. The dashed line shows the time when the perturbation is applied. (c) Time delay calculated at $50 \%$ of the rising edges as a function of distance from the point where the pulse is born. Parameter values are $G_{0}=0.057, q_{0}=3.37, F_{i}=6.05 \times 10^{-2}, A=0.8$, $r_{1}=r_{2}=-0.9, B=0, \alpha=3, \gamma_{t h}=3.5 \times 10^{-2}, L_{G}^{2} / L_{t h}^{2}=$ 0.25 . The perturbation amplitude is $5 \times 10^{-2} . R=40$. 
and transmitted fields, which are found to be

$$
\begin{gathered}
F_{r}=\frac{t_{1} e^{i q z_{0}} F+\left(r_{1}^{\prime}-e^{i 2 q z_{0}}\right) F_{i}}{1+r_{1} e^{i 2 q z_{0}}}, \\
F_{t}=\frac{t_{2} e^{i q\left(L-z_{0}\right)}}{1+r_{1} e^{i 2 q z_{0}}} F .
\end{gathered}
$$

Regarding the field and gain dynamics, our model is equivalent to that in [25] when the optical field is adiabatically eliminated. The main differences are that (i) in our case we consider a local energy balance for the optical power dissipated in the device $H$, while in [25] only the spontaneous emission contribution $B G$ was accounted for, and (ii) that we neglect the temperature dependence of the parameters describing the susceptibility of the active medium since we are assuming that the temperature variations in the QW plane are relatively small.

The numerical integration of the model reproduces the essential qualitative features observed in the experiment. A transition from a low-power state with almost uniform intensity profile to an inhomogeneous high-power state is observed as $G_{0}$ or the injected frequency increase. The inhomogeneity of the high-power state is due to the Turing instability of the optical field that leads to pattern or soliton formation in this system. In between these two states, a localized self-oscillatory regime appears.

When in the low-power state, the system displays localized excitable response to global pulses in $G_{0}$, and propagation effects appear [see Figs. 4(a) and 4(b)]: the initially narrow pulse (see time $t_{1}$ ) broadens as it propagates to the outer regions (times $t_{2}$ and $t_{3}$ ), and the death of the excitable pulse also begins at the center $\left(t_{4}\right)$ and then it propagates away. Moreover, the amplitude of the pulse diminishes as the outer region is approached. This indicates a spatially varying excitability threshold - due to the nonuniformity of the optical intensity induced by the modulational instability - which confines the excitable wave. Since the propagation velocity of an excitable wave depends on the excitability threshold of the system, the delay with respect to the central point in the outer region should then saturate, as observed in the numerical results [see Fig. 4(c)] and also in the experiment [Fig. 3(b)]. The initial slow increase of the delays [first three points in Fig. 4(c)] is due to the fact that in these points the excitable wave is still rising; this effect is not clearly visible in the experiment due to the low spatial resolution of the measurement. Finally, we would like to remark that by neglecting the diffraction term $\left(\nabla_{\perp}^{2} F=0\right)$, i.e., without the aforementioned modulational instability, the excitable waves are not confined and they propagate through the whole space as in a common reaction-diffusion system.

In conclusion, our experiment gives direct evidence of excitable propagating phenomena in semiconductor microcavities. These excitable waves, however, become selfconfined to a finite region of the transverse plane due to an effective spatial variation of the excitability threshold arising from the modulational instability of the optical field in the high-power state. Thus, our results provide a connection with the observed biological and chemical waves while extending previous studies to the case of a modulationally unstable upper state. The connection between selfconfined excitable waves and cavity solitons will be the subject of future experimental and theoretical studies.

We acknowledge financial support from MCYT (Spain) through project TIC2002-04255-C04-03 and European project VISTA. We also thank F. Sagues for helpful discussions and Ulm University for providing the VCSOAs.

[1] J.D. Murray, Mathematical Biology (Springer-Verlag, Berlin, 1989); T. Frisch, S. Rica, P. Coullet, and J. M. Gilli, Phys. Rev. Lett. 72, 1471 (1994); M. Giudici, C. Green, G. Giacomelli, U. Nespolo, and J.R. Tredicce, Phys. Rev. E 55, 6414 (1997).

[2] A. M. Zhabotinskii and A. Zaikin, Nature (London) 225, 535 (1970).

[3] A. T. Winfree, Science 175, 634, (1972).

[4] V. Castets, E. Dulos, J. Boissonade, and P. De Kepper, Phys. Rev. Lett. 64, 2953 (1990).

[5] A. M. Turing, Philos. Trans. R. Soc. London B 237, 37 (1952).

[6] J. Lechleiter, S. Girad, E. Peralta, and D. Clapham, Science 252, 123, (1991).

[7] S. Kondo and R. Asai, Nature (London) 376, 765 (1995).

[8] J. Kenener and J. Snyder, Mathematical Physiology (Springer, New York, 1998).

[9] J. M. Davidenko et al., Nature (London) 355, 349 (1992).

[10] V. Y. Sidorov et al., Phys. Rev. Lett. 91, 208104 (2003).

[11] Chemical Waves and Patterns, edited by R. Kapral and K. Showalter (Kluwer, Dordrecht, Netherlands, 1995).

[12] Yu. Rzhanov, H. Richardson, A. Hagberg, and J. Moloney, Phys. Rev. A 47, 1480 (1993).

[13] P. Coullet, D. Daboussy, and J. R. Tredicce, Phys. Rev. E 58, 5347 (1998).

[14] W. Lu, D. Yu, and R. G. Harrison, Opt. Lett. 24, 578 (1999).

[15] S. Barland, O. Piro, S. Balle, M. Giudici, and J. Tredicce, Phys. Rev. E 68, 036209 (2003).

[16] F. Marino, G. Catalan, P. Sanchez, S. Balle, and O. Piro, Phys. Rev. Lett. 92, 073901 (2004).

[17] R. FitzHugh, Biophys. J. 1, 445 (1961).

[18] M. Brambilla, L. Lugiato, F. Prati, L. Spinelli, and W. Firth, Phys. Rev. Lett. 79, 2042 (1997).

[19] L. Spinelli, G. Tissoni, M. Brambilla, F. Prati, and L. A. Lugiato, Phys. Rev. A 58, 2542 (1998).

[20] S. Barland et al., Nature (London) 419, 699 (2002).

[21] L. Yang and I. R. Epstein, Phys. Rev. Lett. 90, 178303 (2003).

[22] F. Marino and S. Balle, Opt. Commun. 231, 325 (2004).

[23] T. Ackemann et al., Opt. Lett. 25, 814 (2000).

[24] J.D. Murray, Mathematical Biology (Springer-Verlag, Berlin, 1989).

[25] L. Spinelli et al., Phys. Rev. A 66, 023817 (2002). 